\title{
Calculator for myocardial infarction risk in patients with type 2 diabetes mellitus
}

Objectives:

Methods:

\section{Results:}

\section{Conclusions:}

To create a software application (a computer program) based on integral assessment of MI risk in patients with type 2 diabetes mellitus with genetic markers taken into account as well as to develop a method for its prediction in this category of patients.

255 Uzbek men with type 2 diabetes mellitus were recruited for the study. Their demographic and medical history data were registered by means of enquiry; anthropometric and physical examinations were performed. Among biochemical parameters, glycated hemoglobin $(\mathrm{HbAlc})$ was measured, lipid profile and coagulation system were analyzed. Functional examination included ECG and echocardiography. To perform integral assessment and predict MI risk we used method for standardization of intensive parameters by Shigan E.N., based on Bayesian probability(1). Relative risk (RR) is the ratio of the probability of an event occurring (for example, developing a disease, being injured) in an exposed group to the probability of the event occurring in a comparison, non-exposed group. As various risk factors and causes underlying them have different power of influence on the formation of diseases, relative risk was found for each factor by dividing maximum value by the minimum one. Standardized intensive parameter was calculated when frequency of a risk factor was divided by the total frequency of a complication in population. Integral assessment of the whole phenomenon is performed by multiplying the value of standardized intensive parameter $(M)$ by relative risk (RR), that is, by means of the following formula: $\quad X=M^{*} R$. In our study a set of MI risk factors in type 2 diabetes mellitus comprised age, duration of type 2 diabetes mellitus, arterial hypertension, obesity (BMI $>25 \mathrm{~kg} / \mathrm{m} 2), \mathrm{MI}$ in family medical history, left ventricular hypertrophy, diabetic nephropathy, alcoholism, HbAlc $\leq 7 \%$, stroke in family medical history, dyslipidemia, hypercoagulation, smoking, ACE gene DD genotype and TCF7L2 gene TT genotype(2). Among Uzbek patients with type 2 diabetes mellitus MI risk is the highest in persons over 70 years of age $(\mathrm{M}=2.972)$, those with diabetes duration of 16-20 years $(M=3.030)$, those with $M I$ in family medical history $(M=5.349)$, those with left ventricular hypertrophy $(M=2.573)$, those with ACE gene DD genotype $(M=2.320)$, those with TCF7L2 gene TT genotype $(M=5.185)$, those with diabetic nephropathy $(\mathrm{M}=2.443)$, with $\operatorname{HbAlc} \leq 7 \%(\mathrm{M}=1.94)$, those with stroke in family medical history $(\mathrm{M}=2.27)$, dyslipidemia $(\mathrm{M}=1.234)$, hypercoagulation $(\mathrm{M}=1.709)$ as well as in alcoholics $(\mathrm{M}=2.70)$ and smokers $(\mathrm{M}=1.234)$. As to relative risk of factors causing MI in Uzbek men with type 2 diabetes mellitus, myocardial infarction in family medical history comes the first (9.20) followed by left ventricular hypertrophy (7.87), age (5.80), ACE gene DD genotype (4.22), arterial hypertension (4.11), diabetes duration (2.86), diabetic nephropathy (2.77), alcohol abuse (2.34), HbAlc $\leq 7 \%$ (2.00), obesity (BMI> $25 \mathrm{~kg} / \mathrm{m} 2)$ (1.88), stroke in family medical history (1.82), dyslipidemia (1.54), TCF7L2 gene TT genotype (1.51), smoking (1.46) and hypercoagulation (1.26).

This type of rating is essential in a physician's practice to categorize MI risk contingent among patients with type 2 diabetes mellitus. Possible ranges of risk for all factors above were determined after calculation of MI risk relative risk parameters. Maximum and minimum values for each factor were summed up; the sum was divided by three corresponding to risk degree of a complication. MI risk range in type 2 diabetes mellitus scored from 41.0 to 155.8. Consequently, the higher a standardized intensive parameter due to effect of set of the factors studied, the higher is MI risk in patients with type 2 diabetes mellitus. It is expedient to divide the whole risk range into subranges which allow categorizing patients by various risk probability for the risk factors known.

Thus, there are three MI risk subranges for patients with type 2 diabetes mellitus.

1.The lowest MI risk subrange scoring 41.0-79.26 is for patients with favorable prognosis and minimum MI risk. 2.Intermediate MI risk subrange scoring from 79.26 to 117.52 is for patients who have higher probability of MI and require focusing of physicians' attention.

3. The highest MI risk subrange scoring from 163.18 to 207.45 is for patients with unfavorable prognosis maximally affected by MI risk factors.

Based on the calculations above a software application (a computer program) titled "Calculator for myocardial infarction risk in Uzbek patients with type 2 diabetes mellitus" was developed (3).

The program activated, all myocardial infarction risk factors with icons "yes" or "no" under each of them appear on a computer screen. Clicking the corresponding icon, "yes" (presence) or "no" (absence) a physician introduces appropriate data for each MI risk factor. Input of data for all MI risk factors completed, scores for MI risk degree (low, moderate or high) can be obtained by clicking the icon "Calculate". Clicking the icon "Recommendations" a physician can get information about the recommendations for MI prevention in Uzbek patients with type 2 diabetes mellitus.

Prediction of MI risk degree in patients with type 2 diabetes mellitus facilitates timely interventions corresponding to MI risk degree. That will help delay or prevent MI, or smooth its course with minimal or no complications.

1.Shigan E.N. Modeling and hardware in assessment and prediction of public health. Moscow. 1983.-60p.

2.Kapralova Yu.A., Rakhimova G.N., Akbarov Z.S., Takhirova F.A., Turdikulova Sh.U. Association of a rs 7903146 polymorphism in TCF7L2 gene with myocardial infarction in T2DM patients in Uzbekistan. European Journal of Human Genetics. Volume 22, Supplement 1, May 2014. Mylan, Italy. P.408.

3. Official Bulletin of Agency on intellectual property of the Republic of Uzbekistan, Tashkent, 2014, 12 (64), p.159. 